\title{
COMPOSIÇÃO FLORÍSTICA DA REGENERAÇÃO NATURAL DE ESPÉCIES ARBÓREAS AO LONGO DE OITO ANOS EM UM FRAGMENTO DE FLORESTA ESTACIONAL SEMIDECIDUAL, EM VIÇOSA, MG ${ }^{1}$
}

\author{
Pedro Higuchi ${ }^{2}$, Maria das Graças Ferreira Reis ${ }^{3}$, Geraldo Gonçalves dos Reis ${ }^{3}$, Antonio Lelis Pinheiro³, \\ Crodoaldo Telmo da Silva ${ }^{2}$ e Carlos Henrique Rodrigues de Oliveira ${ }^{2}$
}

\begin{abstract}
RESUMO - A florística da regeneração natural foi estudada em 10 locais, com seis sub-parcelas em cada local, em 1992, 1995 e 2000, em fragmento de Floresta Estacional Semidecidual Montana, em Viçosa, MG. A amostragem da classe 1 de tamanho de planta (até $1 \mathrm{~m}$ de altura), classe 2 (1-3 m de altura) e classe 3 ( $>3 \mathrm{~m}$ de altura e $<5 \mathrm{~cm}$ de DAP) foi realizada em unidades de $0,5 \times 10,0,5 \times 20$ e 1 x $20 \mathrm{~m}$, respectivamente. Foram amostrados 4.149 indivíduos pertencentes a 42 famílias e 160 espécies, sendo $11,7 \%$ pioneiras, $62,1 \%$ secundárias iniciais e 26,2\% tardias. O número de famílias permaneceu estável, e o número de espécies aumentou entre 1992 e 2000. Fabaceae, Myrtaceae, Rubiaceae e Lauraceae destacaram-se em todas as classes de tamanho de planta. O número de ingressos foi superior ao desaparecimento de espécies em 1995 e 2000. O Índice de ShannonWeaver foi significativamente $(\mathrm{P} \leq 0,05)$ inferior na classe 1 apenas em 1992, e, considerando a média de todas as classes, aumentou de 3,45 em 1992 para 3,67 e 3,64 em 1995 e 2000, respectivamente. O índice de equabilidade de Pielou por ano de amostragem variou de 0,71 a 0,74 e aumentou de 0,71 na classe 1 , a 0,79 e 0,82 , nas classes 2 e 3 de tamanho. Os resultados do presente estudo indicaram a necessidade de se monitorar a composição florística da regeneração natural, ao longo de um período, em parcelas permanentes, para facilitar o entendimento da dinâmica da vegetação em fragmentos de florestas secundárias.
\end{abstract}

Palavras-chave: Regeneração natural, Floresta Estacional Semidecidual Montana, fragmentos florestais e composição florística

\section{FLORISTIC COMPOSITION OF NATURAL REGENERATION OF TREE SPECIES IN A MOUNTAIN SEASONAL SEMIDECIDUOUS FOREST FRAGMENT OVER AN EIGHT YEAR PERIOD, IN VICGOSA, MG, BRAZIL}

\begin{abstract}
The floristic composition of the natural regeneration in a secondary Seasonal Semideciduous Forest fragment in the Atlantic Forest domain, Southeastern Brazil, was studied in the years 1992, 1995 and 2000. The sampling unit sizes were $0.5 \times 10 \mathrm{~m}, 0.5 \times 20 \mathrm{~m}$ and $1 \times 20 \mathrm{~m}$, respectively, for plant size class 1 (height up to $1 \mathrm{~m}$ ), class 2 (height from 1 to $3 \mathrm{~m}$ ) and class 3 (height $>3 \mathrm{~m}$ and $\mathrm{DBH}<5 \mathrm{~cm}$ ). A total of 4,149 individuals were sampled over the inventory period, comprising 42 families and 160 species, being $11.7 \%$ pioneer, $62.1 \%$ early secondary, and $26.2 \%$ late secondary species. Family number did not change and number of species increased with time. Fabaceae, Myrtaceae, Rubiaceae and Lauraceae were the predominant families. Species ingrowth was higher than mortality. Shannon-Weaver diversity index was significantly lower
\end{abstract}

\footnotetext{
${ }^{1}$ Recebido em 25.02.2005 e aceito para publicação em 13.09.2006.

${ }^{2}$ Programa de Pós-Graduação em Ciência Florestal da UFV, 36570-000 Viçosa - MG, Brasil

${ }^{3}$ Departamento de Engenharia Florestal da UFV, 36570-000 Viçosa, MG, Brasil. E-mail: <mgfreis@ ufv.br>. Bolsistas CNPq.
} 
$(P \leq 0.05)$ only for the plant size class 1 as compared to the other size classes, in 1992, and it increased significantly from 3.45 (average of all plant size classes), in 1992, to 3.67 and 3.64 in 1995 and 2000, respectively. The Pielou equability index per inventory date varied from 0.71 to 0.74 , and it was $0.71 ; 0.79$ and 0.82 for the size classes 1, 2 and 3, respectively. Overall, the results showed that monitoring the natural regeneration over an age sequence is important to understand the dynamics of the secondary forest fragments.

Keywords: Natural regeneration, Semideciduous Seasonal Forest, forest fragment and floristic composition.

\section{INTRODUÇÃO}

As florestas tropicais são de grande importância para a sociedade, principalmente quando são utilizados planos de manejo que possibilitem a exploração sustentável de seus recursos madeireiros e nãomadeireiros, de modo que continuem desempenhando o seu papel nos ciclos hidrológico e do carbono, bem como na manutenção da biodiversidade. Porém, a expansão da fronteira agrícola e a exploração indiscriminada de madeira têm comprometido sua existência, sendo que, por exemplo, na área de domínio da Floresta Atlântica, restaram fragmentos de reduzido tamanho e, às vezes, bastante isolados (PEREIRA et al., 2001). Esses fragmentos se encontram altamente degradados, em razão da exploração seletiva de madeira e do constante pastoreio no seu interior, dentre outros, havendo necessidade de se estabelecerem planos de manejo para a recuperação dessa vegetação (ALMEIDA JÚNIOR, 1999).

A partir da década de 1990, grande número de estudos tem sido desenvolvido sobre a composição florística e a estrutura do estrato arbóreo em fragmentos da Floresta Estacional Semidecidual na Zona da Mata de Minas Gerais, destacando-se os trabalhos de Almeida e Souza (1997), Fernandes (1998), Almeida Junior (1999), Louzada (2002), Paula et al. (2002), Meira-Neto e Martins (2002), Ribas et al. (2003) e Silva et al. (2004). Trabalhos envolvendo a regeneração natural de espécies arbóreas nessa região são raros na literatura, mas essenciais para a compreensão dos processos ecológicos requeridos para o manejo adequado desses fragmentos florestais (VOLPATO, 1994; PEZZOPANE, 2001). Dentre os estudos fitossociológicos sobre a vegetação adulta ou regeneração natural desenvolvidos na Zona da Mata de Minas Gerais, os levantamentos florísticos em seqüência de idade, que permitem melhor entender a dinâmica da sucessão vegetal, são ainda escassos, destacando-se os trabalhos de Paula et al. (2002), Ribas et al. (2003) e Silva et al. (2004).
O presente estudo teve a finalidade de analisar a composição florística da regeneração natural, em um período de oito anos, de um fragmento de Floresta Estacional Semidecidual Montana secundária, em Viçosa, Minas Gerais, com o objetivo de fornecer subsídios à compreensão dos processos ecológicos envolvidos na sucessão secundária.

\section{MATERIAL E MÉTODOS}

O presente estudo foi desenvolvido na Estação de Pesquisa, Treinamento e Educação Ambiental Mata do Paraíso (EPTEA), localizada no Município de Viçosa, MG (20 $45^{\prime}$ Sul, $42^{\circ} 55^{\prime}$ Oeste), com altitude variando de 750 a $850 \mathrm{~m}$. O clima da região é $\mathrm{Cwb}$, de acordo com Köppen, com verão chuvoso e inverno seco, precipitação média anual de $1.221 \mathrm{~mm}$ e temperatura média de $19^{\circ} \mathrm{C}$. No topo dos morros predomina o Latossolo Vermelho-Amarelo, nas encostas das elevações o Argissolo Vermelho-Amarelo, nos terraços o Argissolo Vermelho-Amarelo Câmbico, e nos vales o Neossolo Flúvico associado aos Gleissolos. A vegetação natural da região é classificada como Floresta Estacional Semidecidual Montana, e a cobertura vegetal atual do fragmento estudado é representada por floresta secundária residual em diversos estádios serais (VELOSO et al., 1991; SILVA et al., 2004). A área estudada não sofreu nenhuma intervenção nos últimos 40 anos (FERNANDES, 1998).

O levantamento da regeneração natural, em três classes de tamanho de planta, foi realizado nos anos de 1992, 1995 e 2000, em parcelas permanentes alocadas por Volpato (1994), em função de características fisiográficas como declividade, exposição e posição topográfica do terreno (Quadro 1). Os dados de transmissividade da radiação fotossinteticamente ativa (t\%) e do índice de área foliar (IAF) foram obtidos, respectivamente, através de sensores lineares e analisador de dossel da LI-COR (PEZZOPANE, 2001). 
Quadro 1 - Declividade (D), exposição (Exp.), posição topográfica (PT), transmissividade da radiação fotossinteticamente ativa (t\%) e índice de área foliar (IAF) dos 10 locais estudados, em um fragmento de Floresta Estacional Semidecidual, na EPTEA, Viçosa, MG

Table 1 -Slope (D), aspect (Exp.), topographic position $(P T)$, photosynthetic active radiation transmissivity $(t \%)$ and leaf area index (IAF) of the studied sites, in a Seasonal Semideciduous Forest fragment, Viçosa, MG, Brazil

\begin{tabular}{cccccc}
\hline Local & $\mathrm{D}(\%)^{1}$ & Exp. $^{1}$ & $\mathrm{PT}^{1}$ & $\mathrm{t}(\%)^{2}$ & $\mathrm{IAF}^{2}$ \\
\hline 1 & 40 & $\mathrm{NE}$ & terço superior & 8,9 & 3,6 \\
2 & 21 & $\mathrm{NE}$ & meia encosta & 6,0 & 4,5 \\
3 & 43 & $\mathrm{NE}$ & terço inferior & 2,7 & 4,9 \\
4 & 80 & $\mathrm{NE}$ & meia encosta & 9,3 & 3,6 \\
5 & 3 & - & baixada & 1,7 & 5,2 \\
6 & 51 & $\mathrm{SO}$ & terço inferior & 1,8 & 5,0 \\
7 & 45 & $\mathrm{SO}$ & meia encosta & 1,6 & 5,2 \\
8 & 20 & $\mathrm{SO}$ & meia encosta & 3,7 & 4,2 \\
9 & 14 & $\mathrm{SO}$ & terço inferior & 2,8 & 5,1 \\
10 & 45 & SO & terço superior & 2,5 & 4,6 \\
\hline
\end{tabular}

Fonte: ${ }^{1}$ Fernandes (1998) e ${ }^{2}$ Pezzopane (2001).

O tamanho das parcelas para a amostragem da classe 1 de tamanho de planta (até $1 \mathrm{~m}$ de altura) foi de $0,5 \times 10 \mathrm{~m}$; para a classe 2 ( 1 a $3 \mathrm{~m}$ de altura) foi de $0,5 \times 20$ me para a classe 3 ( $>3 \mathrm{~m}$ de altura $\mathrm{e}<5 \mathrm{~cm}$ de DAP), de 1 x $20 \mathrm{~m}$. Essas parcelas de amostragem da regeneração natural foram alocadas no centro de cada uma das seis parcelas contíguas de 10 x 20 m, instaladas em cada um dos 10 locais para o levantamento da vegetação arbórea adulta, totalizando 60 unidades amostrais para cada classe de tamanho de planta. Assim, a área total de amostragem foi de 300, 600 e $1.200 \mathrm{~m}^{2}$ para as classes 1,2 e 3 , respectivamente.

Os indivíduos amostrados foram reconhecidos em nível de família, gênero e espécie. Os materiais botânicos não reconhecidos no campo, quando possível, foram coletados e identificados no Herbário do Setor de Dendrologia do Departamento de Engenharia Florestal e do Setor de Botânica do Departamento de Biologia Vegetal da Universidade Federal de Viçosa. As espécies foram classificadas pelo sistema do Angiosperm Phylogeny Group II (APG II, 2003).

As espécies foram classificadas em pioneiras, secundárias iniciais e secundárias tardias, com base em revisão de trabalhos realizados na região (PEZZOPANE, 2001; LOUZADA, 2002; SILVA et al., 2004), que utilizaram o sistema de Budowski (1965). Foram utilizados, também, dados do presente estudo para aperfeiçoar a classificação mencionada nesses trabalhos. Por exemplo, uma espécie que tivesse sido classificada como pioneira e secundária inicial por outros autores seria considerada secundária inicial caso ocorresse nas três classes de tamanho de planta da regeneração natural, no presente estudo. Para as espécies identificadas apenas em nível de gênero foi observada a classificação de outras espécies do mesmo gênero, bem como a sua presença nas diferentes classes de tamanho de planta.

A análise da composição florística foi realizada com base na listagem por família e espécie, por classe de tamanho de plantas, nas três amostragens (1992, 1995 e 2000), para o fragmento como um todo. Para a análise da diversidade das espécies arbóreas, foram calculados o índice de diversidade de Shannon-Weaver $\left(H^{\prime}\right)$ e o índice de eqüabilidade de Pielou $(J)$ (SOUZA et al., 2002b) por ano de amostragem e por classe de tamanho de planta, dentro de cada ano de amostragem. As diferenças entre os valores de $H^{\prime}$ foram comparados aos pares, pelo teste t, de Hutcheson (ZAR, 1996), comparando os anos de amostragem e as classes de tamanho de planta dentro de cada ano de amostragem.

\section{RESULTADOS E DISCUSSÃO}

No período de realização deste estudo (1992-2000) foram amostrados 4.149 indivíduos, pertencentes a 42 famílias, 101 gêneros e 160 espécies arbóreas, nos 10 locais estudados e nas três classes de tamanho de planta, sendo que 23 espécies foram identificadas apenas em nível de gênero, 11 em nível de família e cinco não foram identificadas (Quadro 2). As plântulas são difíceis de serem identificadas em razão de não possuírem partes como os órgãos reprodutivos ou, mesmo, características do tronco que ajudam no processo de reconhecimento das espécies, razão de algumas identificações terem sido realizadas apenas em nível de família ou gênero. Para a vegetação adulta desse mesmo local de estudo foi possível a identificação de $84 \%$ das espécies em nível de espécie (SILVA et al., 2004).

Em 1995, três anos após a primeira avaliação houve o ingresso de 16 espécies, a saber: Astrocaryum aculeatissimum (SI), Bignoniaceae 1, Carpotroche brasiliensis (SI), Cassia ferruginea (SI), Desconhecida 2, Desconhecida 3, Hirtella heblecada (ST), Machaerium sp. (P), Melastomataceae 1, Rubiaceae 1, Sapotaceae 1, Swartzia myrtifolia (ST), Tibouchina granulosa (SI), Tovomita glazioviana (SI), Trichilia sp. (ST),

R. Árvore, Viçosa-MG, v.30, n.6, p.893-904, 2006 
Trichilia pallida (ST), predominantemente espécies secundárias iniciais (4) e tardias (4). Nesse mesmo período desapareceram as espécies Alchornea glandulosa $(\mathrm{P})$, Guarea kunthiana (ST), Mabea fistulifera (P), Maprounea guianensis (SI), Ocotea villosa (SI) e Platypodium elegans (SI), sendo duas pioneiras, três secundárias iniciais e uma secundária tardia. No ano de 2000, houve ingresso de 13 espécies em relação a 1995: Acacia glomerosa (SI), Anacardiaceae 1, Eriotheca candolleana (SI), Hymenaea aurea (ST), Mabea fistulifera (P), Maclura tinctoria (SI), Miconia cubatanensis (SI), Myrsinaceae 1, Myrtaceae 1, Ocotea dispersa (SI), Solanum leucodendron (P), Solanum sp. (P) e Tabebuia sp. (SI), predominantemente espécies pioneiras (3) e secundárias iniciais (5). Contudo, nesse mesmo período as seguintes espécies desapareceram: Tapirira obtusa (P), Cecropia hololeuca (P), Terminalia sp. (ST), Desconhecida 3, Sloanea sp. (ST), Trichilia elegans (ST) e Sapotaceae 1, ou seja, duas espécies pioneiras e três secundárias tardias. $\mathrm{O}$ aparecimento de espécies pioneiras em estádios intermediários ou avançados de sucessão se deve, principalmente, à abertura de clareiras devido à morte de árvores de grande porte. No processo de regeneração natural, o ingresso de novas espécies durante determinado ano pode ocorrer ou não devido, dentre outros, à produção irregular de frutos, presença de predadores e, ou, condições ambientais que interferem na germinação das sementes.

Quadro 2 - Espécies arbóreas amostradas em 1992, 1995 e 2000, em ordem alfabética de famílias, gêneros e espécies, nas três classes de tamanho de plantas da regeneração natural $(1,2,3)$ e respectivos grupos ecológicos (GE), classificadas como pioneiras (P), secundárias iniciais (SI) e secundárias tardias (ST), em um fragmento de floresta estacional semidecidual, na EPTEA, Viçosa, MG

Table 2 - Tree species sampled in 1992, 1995 and 2000 in alphabetical order of families, genera and species for each natural regeneration plant size class $(1,2,3)$, followed by their respective ecological group $(P$ - pioneer, SI initial secondary and ST - late secondary), in a Seasonal Semideciduous Forest fragment, Viçosa,MG, Brazil

\begin{tabular}{|c|c|c|c|c|c|c|c|c|c|c|c|}
\hline \multirow[t]{3}{*}{$\overline{\text { Família }}$} & \multirow[t]{3}{*}{ Espécie } & \multirow[t]{3}{*}{$\mathrm{GE}^{1}$} & \multicolumn{3}{|c|}{1992} & \multicolumn{3}{|c|}{1995} & \multicolumn{3}{|c|}{2000} \\
\hline & & & \multicolumn{9}{|c|}{ Classe de tamanho de planta } \\
\hline & & & 1 & 2 & 3 & 1 & 2 & 3 & 1 & 2 & 3 \\
\hline \multirow[t]{2}{*}{$\overline{\text { Anacardiaceae }}$} & Anacardiaceae 1 & & - & - & - & - & - & - & - & + & - \\
\hline & Tapirira guianensis Aublet & $\mathrm{P}$ & + & + & - & + & + & - & + & - & - \\
\hline \multirow[t]{4}{*}{ Annonaceae } & Annona cacans Warm. & SI & + & - & - & + & + & - & + & + & - \\
\hline & Guatteria sellowiana Schlecht. & SI & + & + & - & + & + & - & + & + & + \\
\hline & Rollinia sylvatica Mart. & SI & + & + & + & + & + & + & + & + & + \\
\hline & Xylopia sericea A. St. Hill & $\mathrm{P}$ & + & + & + & + & + & - & + & + & + \\
\hline \multirow[t]{2}{*}{ Apocynaceae } & Aspidosperma parvifolium DC. & SI & + & + & - & - & + & - & + & - & - \\
\hline & Aspidosperma ramiflorum Muell. Arg. & ST & - & + & + & - & + & + & - & + & + \\
\hline Araliaceae & Schefflera morototoni (Aublet) D. Frodin & SI & - & - & + & - & - & + & - & - & + \\
\hline \multirow[t]{4}{*}{ Arecaceae } & Astrocaryum aculeatissimum (Scotht) Burret & SI & - & - & - & + & - & - & + & - & - \\
\hline & Euterpe edulis Mart. & ST & + & + & - & + & + & - & + & + & - \\
\hline & Arecaceae 1 & & + & + & - & + & - & - & + & - & - \\
\hline & Syagrus romanzoffiana (Cham.) Glassm. & SI & + & - & - & + & - & + & + & + & - \\
\hline Asteraceae & Vernonanthura diffusa (Less.) H.Robinson & $\mathrm{P}$ & + & + & + & + & + & + & + & - & - \\
\hline \multirow[t]{6}{*}{ Bignoniaceae } & Bignoniaceae 1 & & - & - & - & + & - & - & + & - & - \\
\hline & Jacaranda macrantha Cham. & SI & + & + & + & + & + & + & + & + & - \\
\hline & Sparattosperma leucanthum K. Schum. & SI & + & - & - & + & - & - & + & + & + \\
\hline & Tabebuia chrysotricha (Mart. ex DC.) Stand. & SI & + & + & - & + & + & + & + & - & + \\
\hline & Tabebuia sp. & SI & - & - & - & - & - & - & + & - & - \\
\hline & Zeyheria tuberculosa (Vell.) Bureau & SI & + & + & - & + & + & - & + & + & + \\
\hline Boraginaceae & Cordia sericicalyx A. DC. & SI & + & + & - & + & + & - & + & - & - \\
\hline Burseraceae & Trattinnickia ferruginea Kuhlm & SI & - & + & + & + & + & + & + & + & + \\
\hline Chrisobalanaceae & Hirtella hebeclada Moric. ex A. P. DC. & ST & - & - & - & + & + & - & + & + & - \\
\hline Clethraceae & Clethra scabra Pers & SI & + & - & - & + & - & - & + & - & - \\
\hline Clusiaceae & $\begin{array}{l}\text { Garcinia gardneriana } \\
\text { (Planchon \& Triana) Zappi. }\end{array}$ & SI & + & + & - & + & + & - & + & + & - \\
\hline
\end{tabular}

R. Árvore, Viçosa-MG, v.30, n.6, p.893-904, 2006 
Quadro 2 - Cont.

Table 2 - Cont.

\begin{tabular}{|c|c|c|c|c|c|c|c|c|c|c|c|}
\hline \multirow[t]{3}{*}{ Família } & \multirow[t]{3}{*}{ Espécie } & \multirow[t]{3}{*}{$\mathrm{GE}^{1}$} & \multicolumn{3}{|c|}{1992} & \multicolumn{3}{|c|}{1995} & \multicolumn{3}{|c|}{2000} \\
\hline & & & \multicolumn{9}{|c|}{ Classe de tamanho de planta } \\
\hline & & & 1 & 2 & 3 & 1 & 2 & 3 & 1 & 2 & 3 \\
\hline \multirow[t]{3}{*}{ Clusiaceae } & Rheedia calyptrata (Schlecht.) & ST & - & + & + & - & - & + & - & - & + \\
\hline & Tovomita glazioviana Engl. & SI & - & - & - & - & + & - & - & + & - \\
\hline & Vismia martiana $\mathrm{H}$. G. Reich & $\mathrm{P}$ & + & + & + & + & + & - & + & + & - \\
\hline Combretaceae & Terminalia sp. & ST & + & + & - & + & + & - & - & + & + \\
\hline Cunoniaceae & Lamanonia ternata Vell. & SI & + & + & + & - & - & + & - & - & + \\
\hline \multirow[t]{5}{*}{ Desconhecidas } & Desconhecida 1 & & - & + & - & + & + & - & + & + & - \\
\hline & Desconhecida 2 & & - & - & - & + & - & - & + & - & - \\
\hline & Desconhecida 3 & & - & - & - & + & - & - & - & - & - \\
\hline & Desconhecida 4 & & + & - & - & + & - & - & - & + & - \\
\hline & Desconhecida 5 & & + & - & - & + & - & - & - & + & - \\
\hline Elaeocarpaceae & Sloanea sp. & ST & - & - & + & - & - & + & - & - & - \\
\hline Erythroxylaceae & Erythroxylum pelleterianum A. St. Hil. & SI & + & - & + & + & + & + & + & + & + \\
\hline \multirow[t]{8}{*}{ Euphorbiaceae } & Alchornea glandulosa Poepp. \& Endl. & $P$ & - & - & + & - & - & - & - & - & - \\
\hline & Alchornea sp. & SI & - & + & - & - & + & - & - & - & - \\
\hline & Aparisthmium cordatum (Juss.) Baill. & SI & + & + & - & + & - & - & + & + & - \\
\hline & Croton floribundus Spreng. & $\mathrm{P}$ & + & + & - & + & + & + & + & + & - \\
\hline & Croton urucurana Baill. & $\mathrm{P}$ & - & + & + & - & - & + & + & - & + \\
\hline & Hyeronima alchorneoides Allem. & SI & + & - & + & + & - & - & - & + & - \\
\hline & Mabea fistulifera Mart. & $\mathrm{P}$ & + & - & + & - & - & - & + & - & - \\
\hline & Maprounea guianensis Aubl. & SI & - & + & + & - & - & - & - & - & - \\
\hline Faboceae & Apuleia leiocarpa Macbride & SI & + & + & + & + & + & + & + & + & + \\
\hline \multirow[t]{9}{*}{ Caesalpinioideae } & Bauhinia forficata Link & $\mathrm{P}$ & + & + & + & + & + & + & + & + & - \\
\hline & Cassia ferruginea Schard. ex DC. & SI & - & - & - & - & + & - & - & + & - \\
\hline & Copaifera langsdorffii Desf. & ST & + & + & - & + & + & - & + & + & - \\
\hline & Hymenaea aurea Desf. & ST & - & - & - & - & - & - & + & - & - \\
\hline & Sclerolobium denundatum Vog. & ST & + & + & + & - & + & + & - & + & + \\
\hline & Senna macranthera (Collad.) Irwin et Barn. & $\mathrm{P}$ & + & + & - & - & + & - & + & - & + \\
\hline & Senna multijuga (Rich.) Irwin et Barn. & $\mathrm{P}$ & + & - & - & + & - & - & - & - & - \\
\hline & Swartzia acutifolia Vog. & ST & + & + & - & + & + & - & + & - & - \\
\hline & Swartzia myrtifolia Smith & ST & - & - & - & + & + & - & + & + & + \\
\hline Faboceae & Andira sp. & ST & + & - & - & + & - & - & + & - & - \\
\hline \multirow[t]{7}{*}{ Faboideae } & Dalbergia nigra Allem. ex Benth. & SI & + & + & + & + & + & + & + & + & + \\
\hline & Machaerium nyctitans (Vell.) Benth. & SI & + & + & + & + & + & + & + & + & + \\
\hline & Machaerium sp. & $\mathrm{P}$ & - & - & - & - & + & - & - & - & - \\
\hline & Machaerium sp.1 & SI & + & - & - & + & + & - & + & + & - \\
\hline & Machaerium stipitatum Vog. & SI & + & + & - & + & + & - & + & + & - \\
\hline & Machaerium triste Vog. & SI & + & + & - & + & + & - & + & + & - \\
\hline & Platypodium elegans Vog. & SI & + & - & - & - & - & - & - & - & - \\
\hline Faboidea & Acacia glomerosa Benth. & SI & - & - & - & - & - & - & + & - & - \\
\hline \multirow[t]{7}{*}{ Mimosoideae } & Anadenanthera peregrina Speg. & SI & + & + & - & + & + & + & + & + & + \\
\hline & Inga edulis Mart. & SI & + & + & + & + & + & + & + & + & + \\
\hline & Inga sp. & SI & - & + & - & - & + & - & - & + & - \\
\hline & Piptadenia gonoacantha Macbride & SI & + & + & + & + & + & + & + & + & + \\
\hline & Pseudopiptadenia contorta (DC.) & SI & + & + & + & + & + & + & + & + & + \\
\hline & Lewis \& P.M. de Lima & & & & & & & & & & \\
\hline & Stryphnodendron guianensis Benth. & SI & + & - & - & + & - & - & + & - & - \\
\hline \multirow[t]{2}{*}{ Icacinaceae } & Citronella paniculata (Mart.) Howard & ST & + & + & + & + & + & + & + & + & - \\
\hline & Villaresia megaphylla Miers & SI & - & + & + & - & + & + & - & + & + \\
\hline
\end{tabular}

R. Árvore, Viçosa-MG, v.30, n.6, p.893-904, 2006 
Quadro 2 - Cont.

Table 2-Cont.

\begin{tabular}{|c|c|c|c|c|c|c|c|c|c|c|c|}
\hline \multirow[t]{3}{*}{ Família } & \multirow[t]{3}{*}{ Espécie } & \multirow[t]{3}{*}{$\mathrm{GE}^{1}$} & \multicolumn{3}{|c|}{1992} & \multicolumn{3}{|c|}{1995} & \multicolumn{3}{|c|}{2000} \\
\hline & & & \multicolumn{9}{|c|}{ Classe de tamanho de planta } \\
\hline & & & 1 & 2 & 3 & 1 & 2 & 3 & 1 & 2 & 3 \\
\hline Lacistemaceae & Lacistema pubescens Mart. & SI & + & + & - & + & + & - & + & + & - \\
\hline \multirow[t]{11}{*}{ Lauraceae } & Endlicheria paniculata (Sprengel) Macbr. & ST & + & - & + & + & - & + & + & + & + \\
\hline & Lauraceae 1 & & + & + & + & + & + & + & + & + & + \\
\hline & Nectandra oppositifolia Nees. & SI & + & + & + & + & + & + & + & + & + \\
\hline & Nectandra megapotamica (Spreng) Mez & ST & + & - & - & + & - & - & - & - & + \\
\hline & Nectandra sp. 2 & ST & + & + & + & + & + & + & + & - & + \\
\hline & Phyllostemonodaphne geminiflora (Mez) Kosterm & SI & - & - & + & + & - & + & + & - & + \\
\hline & Ocotea diospyrifolia (Meissn.) Mez & ST & + & + & - & + & + & + & + & + & + \\
\hline & Ocotea dispersa $\mathrm{Mez}$ & SI & - & - & - & - & - & - & + & - & - \\
\hline & Ocotea villosa Kosterm & SI & + & - & - & - & - & - & - & - & - \\
\hline & Ocotea laxa Mez & SI & + & + & + & - & + & + & + & + & + \\
\hline & Ocotea odorifera (Vell.) Rohwer & ST & - & + & + & + & + & + & + & + & + \\
\hline Lecythidaceae & Cariniana estrellensis Kuntze & ST & + & - & - & + & + & - & + & - & - \\
\hline \multirow[t]{3}{*}{ Malvaceae } & Eriotheca candolleana (K. Schum.) A. Robyns & SI & - & - & - & - & - & - & - & - & - \\
\hline & Luehea grandiflora Mart. \& Zucc. & SI & + & + & + & + & + & + & + & + & + \\
\hline & Pseudobombax longiflorum Mart. et Zucc. & SI & + & + & + & - & - & + & - & - & + \\
\hline \multirow[t]{5}{*}{ Melastomataceae } & Melastomataceae 1 & & - & - & - & + & + & - & + & + & - \\
\hline & Miconia candolleana Triana & SI & + & + & + & + & + & + & + & + & + \\
\hline & Miconia cubatenensis Hoehne & SI & - & - & - & - & - & - & + & - & - \\
\hline & Miconia latecrenata Naud. & SI & + & + & + & - & + & + & + & + & + \\
\hline & Tibouchina granulosa Cogn. & SI & - & - & - & + & + & - & + & - & - \\
\hline \multirow[t]{7}{*}{ Meliaceae } & Cabralea canjerana (Vell.) Mart. & ST & + & + & - & - & + & - & - & + & - \\
\hline & Guarea kunthiana A. Juss. & ST & + & + & - & - & - & - & - & - & - \\
\hline & Guarea trichilioides $\mathrm{L}$. & SI & + & + & + & + & + & + & + & + & + \\
\hline & Guarea pendula R.S. Ramalho et. al & SI & - & + & - & + & + & - & + & + & - \\
\hline & Trichilia elegans Arjuss. & ST & + & + & - & - & + & - & - & - & - \\
\hline & Trichilia sp. & ST & - & - & - & + & - & - & - & + & - \\
\hline & Trichilia pallida Mart. & ST & - & - & - & + & - & - & - & + & - \\
\hline \multirow[t]{3}{*}{ Moraceae } & Brosimum guianense Huber ex Ducke & ST & + & + & + & + & + & + & + & - & + \\
\hline & Maclura tinctoria D. Don ex Stend. & SI & - & - & - & - & - & - & + & - & - \\
\hline & Sorocea bonplandii (Baill.) Burguer, Lana. & SI & + & + & + & + & + & + & + & + & + \\
\hline \multirow[t]{2}{*}{ Myrcinaceae } & Myrsine ferruginea (Mez) Pipoly & SI & + & - & - & + & + & - & + & + & + \\
\hline & Myrsinaceae 1 & & - & - & - & - & - & - & + & - & - \\
\hline Myristicaceae & Virola oleifera (Schott) A. C. Sm. & SI & + & - & + & - & - & + & - & - & + \\
\hline \multirow[t]{11}{*}{ Myrtaceae } & Calyptranthes sp. & ST & + & + & - & + & + & - & + & + & - \\
\hline & Eugenia brasiliensis Lam. & SI & + & + & + & + & + & + & + & + & + \\
\hline & Eugenia cerasiflora Miq. & SI & + & + & + & + & + & + & + & + & + \\
\hline & Eugenia leptoclada Berg. & SI & + & - & - & + & - & + & + & + & + \\
\hline & Eugenia sp. & SI & - & + & - & - & + & - & - & + & - \\
\hline & Myrcia fallax DC. & SI & + & + & + & + & + & + & + & + & + \\
\hline & Myrcia sp. 1 & SI & + & - & - & + & + & + & + & + & + \\
\hline & Myrcia sp. 2 & SI & - & + & - & - & + & - & - & + & - \\
\hline & Myrtaceae 1 & & - & - & + & - & - & + & + & - & - \\
\hline & Myrtaceae 2 & & - & - & - & - & + & - & - & + & - \\
\hline & Psidium guajava $\mathrm{L}$. & ST & + & + & - & - & + & - & - & + & + \\
\hline \multirow[t]{2}{*}{ Nyctaginaceae } & Guapira opposita(Vell.) Reitz & SI & + & + & + & + & + & + & + & + & + \\
\hline & Torrubia schmidtiana (Hemerl.) Standl. & SI & - & + & - & + & + & - & + & + & - \\
\hline \multirow[t]{2}{*}{ Ochnaceae } & Ouratea polygyna Engl. & SI & - & + & - & - & + & - & + & + & - \\
\hline & Ouratea sp. & SI & - & + & - & - & + & - & + & + & + \\
\hline
\end{tabular}

R. Árvore, Viçosa-MG, v.30, n.6, p.893-904, 2006 
Quadro 2 - Cont.

Table 2 - Cont

\begin{tabular}{|c|c|c|c|c|c|c|c|c|c|c|c|}
\hline \multirow[t]{3}{*}{ Família } & \multirow[t]{3}{*}{ Espécie } & \multirow[t]{3}{*}{$\mathrm{GE}^{1}$} & \multicolumn{3}{|c|}{1992} & \multicolumn{3}{|c|}{1995} & \multicolumn{3}{|c|}{2000} \\
\hline & & & \multicolumn{9}{|c|}{ Classe de tamanho de planta } \\
\hline & & & 1 & 2 & 3 & 1 & 2 & 3 & 1 & 2 & 3 \\
\hline Rosaceae & Prunus mystifolia (L.) Urb & ST & + & + & + & + & + & + & + & + & + \\
\hline \multirow[t]{9}{*}{ Rubiaceae } & Coutarea hexandra (Jacquin) Schuman & SI & - & - & + & - & + & - & - & + & - \\
\hline & Ixora venulosa Muell. Arg. & ST & - & + & - & - & + & - & + & + & - \\
\hline & Landenbergia hexandra Klotzsch & ST & + & + & + & + & + & + & + & + & + \\
\hline & Psychotria conjungens Muell. Arg. & ST & + & + & - & + & + & - & + & + & + \\
\hline & Psychotria sessilis (Vell.) Mull. Arg. & SI & + & + & + & + & + & + & + & + & + \\
\hline & Psychotria sp. & SI & + & + & - & + & + & - & + & + & - \\
\hline & Randia nitida (Kunth.) DC. & SI & - & + & + & - & + & + & + & + & + \\
\hline & Rubiaceae 1 & & - & - & - & + & - & - & + & + & - \\
\hline & Rubiaceae 2 & & + & + & - & + & + & - & + & + & - \\
\hline \multirow[t]{4}{*}{ Rutaceae } & Dictyoloma vandellianum A. Juss. & SI & + & + & - & + & + & + & + & + & - \\
\hline & Zanthoxylum sp. & SI & + & - & - & - & - & + & - & - & + \\
\hline & Zanthoxylum rhoifolium Lam. & ST & + & + & + & + & + & + & + & + & + \\
\hline & Zanthoxylum riedelianum Engl. & SI & + & + & + & + & + & + & + & + & + \\
\hline \multirow[t]{4}{*}{ Salicaceae } & Carpotroche brasiliensis Endl. & SI & - & - & - & + & - & - & + & - & - \\
\hline & Casearia aculeata Jacq. & SI & + & - & + & + & + & + & + & + & + \\
\hline & Casearia arborea (L.C. Rich.) Urban. & SI & + & - & - & + & - & - & + & - & - \\
\hline & Casearia gossypiosperma Briq. & SI & + & - & + & - & + & + & + & + & + \\
\hline \multirow[t]{6}{*}{ Sapindaceae } & Allophylus edulis Radlk. Ex Warm. & SI & + & + & - & + & + & - & + & + & + \\
\hline & Allophylus sericeus (Cambess.) Radlk. & ST & + & - & - & + & - & - & + & - & - \\
\hline & Cupania sp. & ST & + & + & + & + & + & + & + & + & + \\
\hline & Cupania vernalis Cambess. & ST & + & + & + & + & - & + & - & - & + \\
\hline & Matayba elaeagnoides Radlk. & SI & + & - & - & + & - & - & + & - & - \\
\hline & Matayba sp. & SI & - & + & + & - & + & + & - & - & - \\
\hline \multirow[t]{3}{*}{ Sapotaceae } & Chrysophyllum flexuosum Mart. & ST & - & - & + & + & + & + & + & - & - \\
\hline & Manilkara sp. & SI & + & - & + & + & + & + & + & - & - \\
\hline & Sapotaceae 1 & & - & - & - & + & - & - & - & - & - \\
\hline Simaroubaceae & Picramnia glazioviana Engler & ST & + & + & + & + & + & + & + & + & + \\
\hline \multirow[t]{2}{*}{ Siparunaceae } & Siparuna guianensis Aubl. & SI & + & + & + & + & + & + & + & + & + \\
\hline & Siparuna sp. & SI & + & + & - & + & + & - & + & + & + \\
\hline \multirow[t]{4}{*}{ Solanaceae } & Solanum argenteum Dun. ex Poir. & $\mathrm{P}$ & + & - & + & - & - & + & - & + & - \\
\hline & Solanum cernuиm Vell. & $\mathrm{P}$ & + & + & + & + & + & - & - & - & + \\
\hline & Solanum leucodendron Sendt & $\mathrm{P}$ & - & - & - & - & - & - & + & - & - \\
\hline & Solanum sp. & $\mathrm{P}$ & - & - & - & - & - & - & - & + & - \\
\hline \multirow[t]{2}{*}{ Urticaceae } & Cecropia hololeuca Miq. & $\mathrm{P}$ & + & - & - & - & + & - & - & - & - \\
\hline & Cecropia pachystachya Trécul & $\mathrm{P}$ & + & - & - & - & - & - & - & - & - \\
\hline \multirow[t]{2}{*}{ Verbenaceae } & Cytharexylum sp. & SI & + & - & - & - & - & - & + & - & - \\
\hline & Vitex sellowiana Cham. & SI & + & + & + & + & + & + & + & + & + \\
\hline
\end{tabular}

Dezesseis espécies secundárias iniciais e seis secundárias tardias ocorreram em todas as classes de tamanho de planta, nos três levantamentos florísticos. Bauhinia forficata e Xylopia sericea, que são pioneiras, não ocorreram em apenas uma das classes de tamanho de planta, em um dos levantamentos, porém apresentaram densidade e freqüência baixas, ocorrendo, possivelmente, em clareiras. As espécies pioneiras, de modo geral, permaneceram nas classes 1 e 2 de tamanho (Solanum argenteum, Tapirira guianensis e Vismia martiana) e, muitas vezes, tiveram vida curta (Alchornea glandulosa, Cecropia hololeuca, Cecropia pachystachya, Mabea fistulifera, Machaerium sp., Senna multijuga, Solanum sp. e Solanum leucodendron). Vernonanthura diffusa, também pioneira, encontravase nas três classes de tamanho em 1992 e 1995, porém, em 1995, os indivíduos das classes 2 e 3 morreram. Euterpe edulis, espécie classificada como secundária tardia, estava presente nas classes 1 e 2, não tendo atingido a classe 3 de tamanho de planta. Essa espécie, 
porém, se encontra na vegetação adulta $($ DAP $>5 \mathrm{~cm})$ e é uma planta de porte mediano (SILVA et al., 2004). Possivelmente, a falta de indivíduos dessa espécie na classe 3 se deve à falta de produção de sementes em algum período anterior ao início desse monitoramento.

Considerando a média dos três levantamentos, apenas $11,7 \%$ das espécies da regeneração natural desse fragmento estudado é constituído por pioneiras, havendo predomínio de secundárias iniciais $(62,1 \%)$, seguidas de tardias $(26,2 \%)$. Na vegetação arbórea adulta desse mesmo fragmento, com base em levantamentos realizados em 1992, 1995, 1998 e 2001, $14,3 \%$ das espécies foram classificadas como pioneiras, $60,9 \%$ como secundárias iniciais e o restante como secundárias tardias (SILVA et al., 2004). É provável que a proporção de pioneiras seja mais elevada na vegetação adulta desse fragmento, em comparação com a regeneração natural, pelo fato de ainda existirem indivíduos desse grupo com elevado porte que se estabeleceram quando o dossel se encontrava mais aberto. Com o fechamento do dossel há o favorecimento da regeneração natural de espécies de estádios mais avançados de sucessão. E, com a morte dos indivíduos de espécies pioneiras, deve ocorrer a sua substituição por espécies secundárias iniciais e tardias que se encontram no banco de plântulas, o que promove redução na proporção de pioneiras na vegetação adulta. A área de estudo apresenta abertura de dossel variável, sendo que o índice de área foliar varia de 3,6 a 5,2 e a transmissividade da radiação fotossinteticamente ativa, de 1,6 a 9,3\% (Quadro 1), demonstrando a grande variabilidade nas condições microclimáticas, que certamente influenciam o estabelecimento das plantas. Possivelmente, a maioria das espécies pioneiras se encontra nas áreas que ainda apresentam dossel mais aberto ou em clareiras que se formam com a queda natural de árvores.

Comparando a lista de espécies do presente estudo com a da vegetação adulta (SILVA et al., 2004), verificase que $71 \%$ das espécies que foram classificadas em nível de espécie na regeneração natural se encontram na lista da vegetação adulta. Considerando que várias espécies da regeneração natural somente foram classificadas no nível de gênero ou família, essa proporção deverá aumentar quando for possível a sua classificação ao nível de espécie. Dentre as espécies da vegetação adulta que não se encontram na regeneração natural, estão incluídas 50,0\% das pioneiras, $39 \%$ das secundárias iniciais e apenas $34 \%$ das tardias, ou seja, maior proporção de espécies pioneiras poderão deixar de fazer parte desse fragmento florestal, sendo, eventualmente, substituídas pelas secundárias, a não ser que façam parte do estoque de sementes do solo. Ao todo, 39\% das espécies arbóreas adultas não estavam representadas na regeneração natural. Possivelmente essas espécies apresentaram número reduzido de indivíduos ou, ainda, não estavam produzindo sementes para garantir a sua continuidade. São, em sua maioria, espécies raras no sistema e, portanto, devem merecer especial atenção quando do estabelecimento de planos de manejo da vegetação para evitar o seu desaparecimento. Há, porém, necessidade de estender este estudo por período mais longo, para testar estas hipóteses. Vale salientar que a área amostral da regeneração natural é menor do que para a vegetação de maior porte, e algumas espécies podem não ter sido amostradas até o presente momento.

A família Fabaceae se destacou na regeneração natural em número de indivíduos e famílias, nas três classes de tamanho de plantas, exceto para número de indivíduos na classe 3, que teve o predomínio de Siparunaceae, principalmente no ano 2000 (Figura 1). O número de indivíduos da família Rubiaceae foi também elevado nas três classes de tamanho de planta, porém o número de espécies dessa família é reduzido na classe 1. No entanto, as famílias Myrtaceae e Lauraceae apresentaram reduzido número de indivíduos com número de espécies mais elevado do que para Rubiaceae, especialmente nas classes 1 e 2. Euphorbiaceae não está entre as cinco famílias com maior número de indivíduos, porém se destaca em relação ao número de espécies. Essas famílias também se destacam no estrato arbóreo adulto desse mesmo fragmento (SILVA et al., 2004).

Quinze famílias apresentaram apenas uma espécie, no presente estudo, sendo elas: Araliaceae, Asteraceae, Boraginaceae, Burseraceae, Chrisobalanaceae, Clethraceae, Combretaceae, Cunoniaceae, Elaeocarpaceae, Erythroxylaceae, Lacistemaceae, Lecythidaceae, Myristicaceae, Rosaceae e Simaroubaceae (Quadro 2). Dentre essas famílias, apenas uma era constituída por espécie pioneira, havendo o predomínio de secundárias iniciais (8) e tardias (6). Silva et al. (2004) constataram que, na vegetação arbórea adulta desse mesmo fragmento, 14 famílias apresentaram apenas uma espécie, sendo que apenas duas eram constituídas de espécies pioneiras, predominando secundárias iniciais (9). 

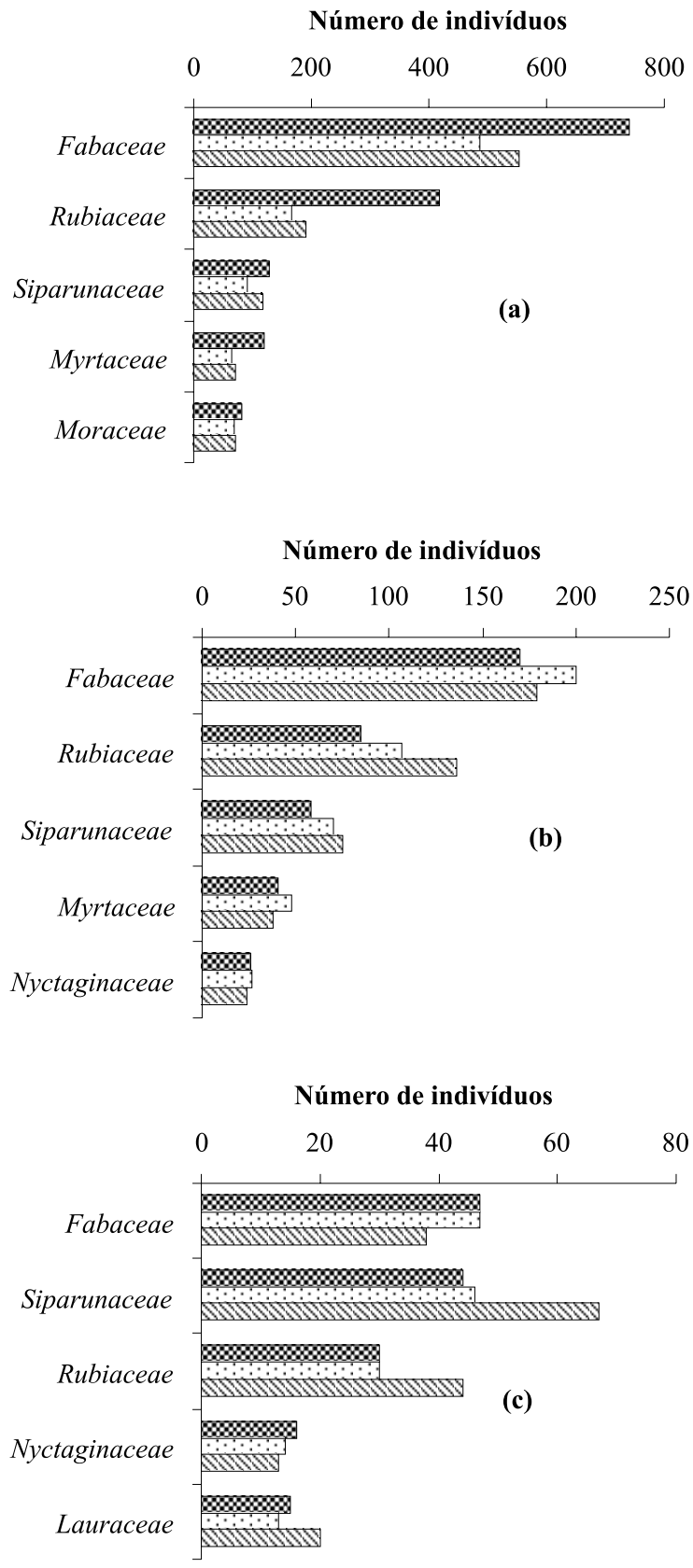
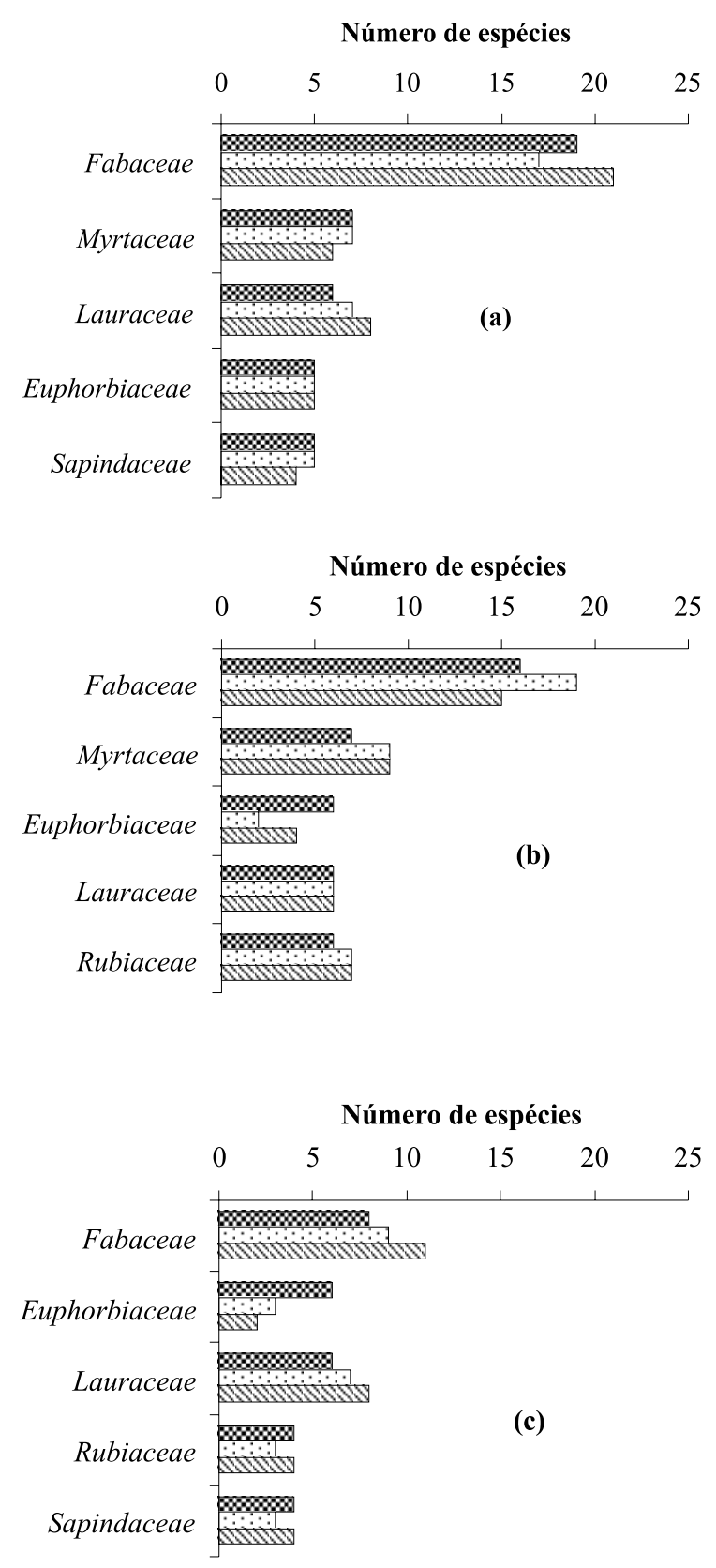

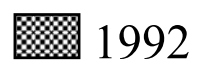

Figura 1 - Números de indivíduos e espécies arbóreas da regeneração natural, por família, nas três classes de tamanho de planta (classe 1 - a; classe 2 - b e classe 3 - c) nos anos de 1992, 1995 e 2000, em um fragmento de Floresta Estacional Semidecidual na EPTEA, Viçosa, MG.

Figure 1 - Numbers of individuals and tree species per different natural regeneration plant size classes (class 1 -a; class $2-b$ and class $3-c)$, and survey dates (1992, 1995 and 2000), in a Seasonal Semideciduous Forest fragment, Viçosa, MG, Brazil. 
O número de famílias permaneceu estável ao longo do período de monitoramento, tendo tido 41, 42 e 41 famílias, respectivamente, em 1992, 1995 e 2000, e o número de espécies aumentou gradativamente de 132 (1992) para 142 (1995) e 149 (2000) (Quadro 2). Para essa mesma área de estudo foram encontradas 146 , 154, 157 e 150 espécies no estrato arbóreo adulto, respectivamente, nos anos de 1992, 1995, 1998 e 2001 (SILVA et al., 2004). Em uma seqüência de levantamento do estrato arbóreo, realizado a cada dois anos, após a eliminação de cipós em uma Floresta Ombrófila Densa, foram encontradas 138, 184, 202, 206 e 273 espécies arbóreas na regeneração natural (SOUZA et al., 2002b). Esses resultados indicaram haver mudança mais acentuada no número de espécies na floresta manejada através da eliminação de cipós. Embora o número de espécies no presente trabalho tenha sido estável ao

(a)

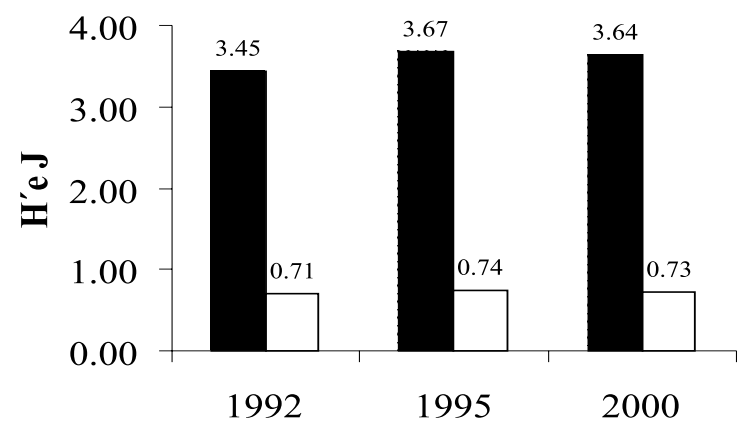

(c)

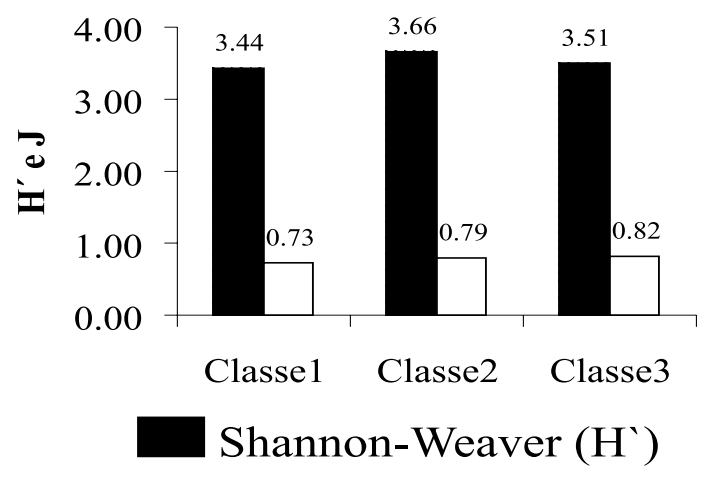

longodoperíododemonitoramentoforamobservadas mudanças na composição florística, conforme discutido anteriormente.

O índice de equabilidade de Pielou (Figura 2) variou apenas entre 0,71 e 0,74 , na análise realizada por ano de amostragem, independentemente da classe de tamanho de planta. Porém, quando o índice foi calculado por classe de tamanho de planta, foi observado que, para a classe 1 , o valor foi 0,71 , para a classe 2 foi 0,79 e para a classe $3,0,82$, ou seja, houve aumento do índice com o aumento da classe de tamanho de planta. Em fragmentos de Floresta Estacional Semidecidual do Alto Rio Grande, o índice de equabilidade de Pielou variou de 0,75 a 0,88 (OLIVEIRA-FILHO et al., 2004), indicando que os resultados de equabilidade do presente estudo se encontram dentro dos limites observados para o mesmo tipo de vegetação em outras regiões.

(b)

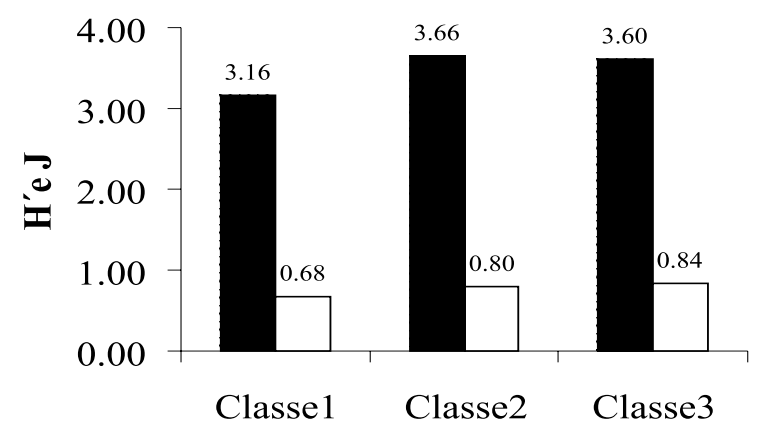

(d)

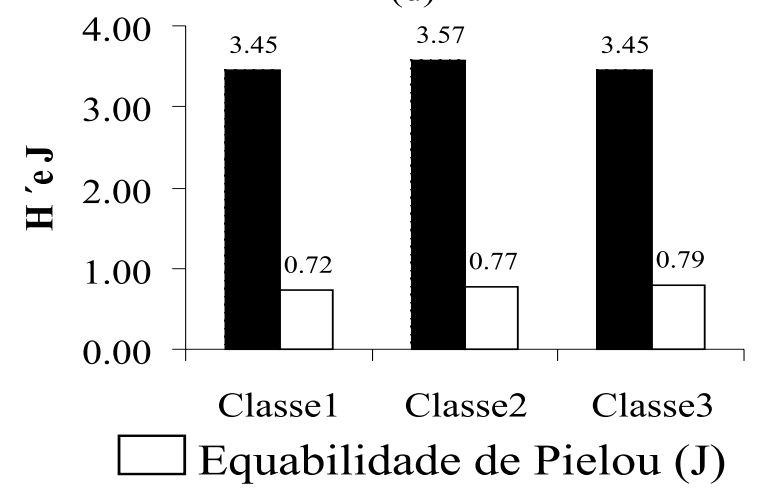

Figura 2 - Índice de Shannon-Weaver e equabilidade de Pielou, por ano de amostragem (a) e por classe de tamanho de plantas nos anos de 1992 (b), 1995 (c) e 2000 (d), em fragmento de Floresta Estacional Semidecidual na EPTEA, Viçosa, MG

Figure 2 - Shannon-Weaver diversity index $\left(H^{\prime}\right)$ and Pielou's equability for all plant size classes (a) and per each natural regeneration plant size class in the years 1992 (b), 1995 (c) and 2000 (d), in a Seasonal Semideciduous Forest fragment, Viçosa, MG, Brazil.

R. Árvore, Viçosa-MG, v.30, n.6, p.893-904, 2006

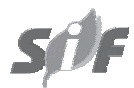


A diversidade de espécies arbóreas na regeneração natural, estimada pelo índice de Shannon-Weaver ( $\left.\mathrm{H}^{\prime}\right)$, foi, em média, 3,59, tendo aumentado significativamente, no nível de 5\% de probabilidade, de 3,45 em 1992 para 3,67 em 1995 e 3,64 em 2000, não tendo sido observado diferença significativa entre 1995 e 2000 (Figura 2 e Quadro 3). Quando o teste t de Hutcheson foi utilizado para avaliar diferenças em H' entre classes de tamanho de planta, para cada ano de amostragem, foi verificado diferença significativa apenas no ano de 1992, sendo H' da classe $1(3,16)$ significativamente inferior ao das classes 2 e $3(3,66$ e 3,60, respectivamente) (Figura 2 e Quadro 3). Os valores para o índice de diversidade do presente estudo foram similares aos obtidos por Louzada (2002), na mesma região, em estudo que envolveu o levantamento de espécies arbóreas acima de $3 \mathrm{~m}$ de altura, em diferentes exposições do terreno, tendo o H' variado de 3,22 a 3,71. Para a vegetação arbórea com diâmetro superior a $10 \mathrm{~cm}$, nas mesmas parcelas do presente estudo, H' variou apenas de 4,20 a 4,23 (SILVA et al., 2004). Para a regeneração natural de floresta ombrófila densa no Espírito Santo, H' aumentou de 4,35 para 5,00, em um período de oito anos, sendo a média de 4,62 (SOUZA et al., 2002b) e, para a vegetação adulta, H' médio foi de 5,05 nessa floresta, não tendo sido observado variação significativa nesse índice ao longo do período de monitoramento (SOUZA et al., 2002a), a exemplo do que foi observado por Silva et al. (2004).

Quadro 3 - Teste t de Hutcheson para o índice de diversidade de Shannon-Weaver (H') nas três classes de tamanho de plantas (1,2,3), nos anos de 1992, 1995 e 2000, emum fragmento de FlorestaEstacional Semidecidual na EPTEA, Viçosa, MG ( $\left.<<0,001 ; \mathrm{t}_{(\mathrm{tab})}=3,32\right)$

Table 3 -Hutcheson t test for the Shannon-Weaver diversity index for different natural regeneration plant size classes and survey date (1992, 1995 and 2000), in a Seasonal Semideciduous Forest fragment, Viçosa, $M G$, Brazil $\left(p<0,001 ; t_{(t a b .)}=3,32\right)$

\begin{tabular}{ccccc}
\hline & & Classe 1 & Classe 2 & Classe 3 \\
\hline \multirow{2}{*}{1992} & Classe 1 & 0,00 & 7,52 & 5,32 \\
& Classe 2 & $*$ & 0,00 & 0,59 \\
& Classe 3 & $*$ & $*$ & 0,00 \\
\multirow{2}{*}{1995} & Classe 1 & 0,00 & 3,28 & 0,75 \\
& Classe 2 & $*$ & 0,00 & 1,58 \\
& Classe 3 & $*$ & $*$ & 0,00 \\
& Classe 1 & 0,00 & 1,71 & 0,01 \\
& Classe 2 & $*$ & 0,00 & 1,18 \\
& Classe 3 & $*$ & $*$ & 0,00 \\
\hline
\end{tabular}

Verificou-se que o índice de diversidade de ShannonWeaver foi mais elevado para a vegetação adulta do que para a regeneração natural, nesses dois estudos, indicando possivelmente que essas áreas se encontravam em estádios mais avançados de sucessão. Em estádios iniciais de sucessão, em que há o predomínio de poucas espécies na vegetação adulta, a regeneração natural pode apresentar diversidade florística mais elevada, em razão das condições microclimáticas sob o dossel das plantas em favorecer o estabelecimento de espécies secundárias iniciais e tardias.

\section{CONCLUSÕES}

O número de famílias permaneceu estável, porém houve aumento no número de espécies no período de monitoramento, o que promoveu aumento na diversidade florística do fragmento, mas sem modificar a proporção de espécies pertencentes aos diferentes grupos ecológicos. A proporção de espécies pioneiras na regeneração natural foi mais baixa do que no estrato da vegetação adulta possivelmente em razão de o dossel já se encontrar fechado, dificultando o estabelecimento de plantas desse grupo ecológico. A proporção de pioneiras do estrato arbóreo que não se encontram no banco de plântulas é mais elevado do que de espécies secundárias, principalmente as tardias. Esses resultados indicam que ao longo de oito anos de estudo o fragmento florestal estudado apresentou algum avanço no seu estádio sucessional, facilitando o estabelecimento de espécies secundárias iniciais e tardias. Havendo necessidade de acelerar o processo de sucessão, alguns indivíduos arbóreos de maior porte, principalmente de espécies pioneiras, podem ser removidos em locais onde tenha banco de plântulas adequado para ocupar rapidamente as novas clareiras.

\section{REFERÊNCIAS BIBLIOGRÁFICAS}

ALMEIDA, D.S.; SOUZA, A.L. Florística e estrutura de um fragmento de Floresta Atlântica, no município de Juiz de Fora, Minas Gerais. Revista Árvore, v. 21, n. 2, p. 221-230, 1997.

ALMEIDA JÚNIOR, J.S. Florística e fitossociologia de fragmentos da floresta estacional semidecidual, Viçosa, Minas Gerais. 1999. 148 f. Dissertação (Mestrado em Ciência Florestal) Universidade Federal de Viçosa, Viçosa, MG, 1999.

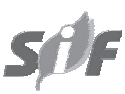

R. Árvore, Viçosa-MG, v.30, n.6, p.893-904, 2006 
ANGIOSPERM PHYLOGENY GROUP-APG II. An update of the Angiosperm Phylogeny Group classification for the orders and families of flowering plants: APG II. Botanical Journal of the Linnean Society, v. 141, p. 399-436, 2003.

BUDOWSKI, G. Distribution of tropical american rain forest species in the light of successional processes. Turrialba, v. 15, n. 1, p. 40-42, 1965.

FERNANDES, H.A.C. Dinâmica e distribuição de espécies arbóreas em uma floresta secundária no domínio da Mata Atlântica. 1998. 151 f. Dissertação (Mestrado em Ciência Florestal) - Universidade Federal de Viçosa, Viçosa, MG, 1998.

LOUZADA, C. Composição florística e estrutura de vegetação arbórea em diferentes condições fisiográficas de um fragmento de floresta estacional semidecidual secundária, na Zona da Mata de Minas Gerais. 2002. 149 f. Dissertação (Mestrado em Ciência Florestal) Universidade Federal de Viçosa, Viçosa, MG, 2002.

MEIRA-NETO, J.A.A.; MARTINS, F.R. Composição florística de uma Floresta Estacional Semidecidual Montana no Município de Viçosa-MG. Revista Árvore, v. 26, n. 4, p. 437-446, 2002.

OLIVEIRA-FILHO, A.T. et al. Variações estruturais do compartimento arbóreo de uma floresta semidecídua alto-montana na Chapada das Perdizes, Carrancas, MG. Revista Brasileira de Botânica, v. 27, n. 2, p. 129-309, 2004.

PAULA, A. et al. Alterações florísticas ocorridas num período de quatorze anos na vegetação arbórea de uma Florestal Estacional Semidecídua em Viçosa-MG. Revista Árvore, v. 26, n. 6, p. 743-749, 2002.

PEREIRA, R. A. et al. Caracterização da paisagem, com ênfase em fragmentos florestais, do município de Viçosa, Minas Gerais. Revista Árvore, v. 25, n. 3, p. 327-333, 2001.
PEZZOPANE, J.E.M. Caracterização fitossociológica, microclimática e ecofisiológica em uma floresta estacional semidecidual secundária. 2001. 225 f. Tese (Doutorado em Ciência Florestal) Universidade Federal de Viçosa, Viçosa, MG, 2001.

RIBAS, R.F. et al. Composição florística de dois trechos em diferentes etapas serais de uma Floresta Estacional semidecidual, em Viçosa, Minas Gerais. Revista Árvore, v. 27, n. 6, p. 821-830, 2003.

SILVA, C.T. et al. Avaliação temporal da florística arbórea de uma floresta secundária no município de Viçosa, Minas Gerais. Revista Árvore, v. 28, n. 3, p. 429-441, 2004.

SOUZA, A.L. et al. Dinâmica da composição florística de uma floresta ombrófila densa secundária, após corte de cipós, Reserva Natural da Companhia Vale do Rio Doce S.A., Estado do Espírito Santo, Brasil. Revista Árvore, v. 26, n. 5, p. 549-558, 2002a.

SOUZA, A.L. et al. Dinâmica da regeneração natural em uma floresta ombrófila densa secundária, após corte de cipós, Reserva Natural da Companhia Vale do Rio Doce S.A., Estado do Espírito Santo, Brasil. Revista Árvore, v. 26, n. 4, p. 411-419, 2002 b.

VELOSO, H.P.; RANGEL FILHO, A.L.R.; LIMA, J.C.A. Classificação da vegetação brasileira, adaptada a um sistema universal. Rio de Janeiro: IBGE, 1991. 123p.

VOLPATO, M.M.L. Regeneração natural em uma floresta secundária no domínio de Mata Atlântica: uma análise fitossociológica. 1994. $123 \mathrm{f}$. Dissertação (Mestrado em Ciência Florestal) Universidade Federal de Viçosa, Viçosa, MG, 1994.

ZAR, J.H. Biostatistical analysis, 3. ed. New Jersey: Prentice Hall, 1996.156-1585 p. 